\title{
A Review of Mitochondrial-derived Fatty Acids in Epigenetic Regulation of Obesity and Type 2 Diabetes
}

\author{
Erin M Taylor, Aarin D Jones and Tara M Henagan* \\ Department of Nutrition Science, Purdue University, West Lafayette, IN, USA
}

Received: July 11, 2014; Accepted: July 28, 2014; Published: August 07, 2014

*Corresponding author: Tara M. Henagan, PhD, Assistant Professor, Department of Nutrition Science, Purdue University, West Lafayette, IN, USA, Tel: 765-494-4536; Fax: 765-494-0906; E-mail: thenagan@purdue.edu

\begin{abstract}
Type 2 diabetes, the leading metabolic disease, is characterized by insulin resistance and is associated with obesity. The onset of type 2 diabetes is largely due to environmental inputs, such as high dietary fat content and decreased levels of exercise. Insulin resistance resulting from high fat diet is associated with skeletal muscle mitochondrial dysfunction, leading to alterations in lipid accumulation and specific species of intracellular fatty acids; whereas, exercise training augments insulin resistance while improving skeletal muscle mitochondrial function and producing beneficial fatty acid profiles. Additionally, high fat diets and exercise alter epigenetic modifications, including DNA methylation and histone acetylation, to produce differences in metabolic gene expression that are associated with insulin resistance and sensitivity, respectively. Recent evidence suggests that short chain fatty acids that act as histone deacetylase inhibitors prevent and ameliorate obesity and insulin resistance. Here, we discuss the potential of mitochondrial-derived fatty acids, especially short chain fatty acids, to epigenetically regulate obesity and type 2 diabetes.
\end{abstract}

Keywords: Obesity; Diabetes; Mitochondria; Fatty acids; Epigenetics

\section{Abbreviations}

HAT: Histone Acetyltransferase; HDAC: Histone Deacetylase

\section{Obesity and Diabetes: Diet and Exercise Effects}

Type 2 diabetes, a disease state characterized by insulin resistance and associated with obesity, is the most common metabolic disorder in the world [1]. Metabolic abnormalities that contribute to the onset and progression of insulin resistance include impaired fatty acid oxidation, ectopic lipid deposition and lipotoxicity [2-5]. A major site of ectopic lipid deposition and arguably one of the first tissues to exhibit insulin resistance during the onset of type 2 diabetes is skeletal muscle [6]. Excess lipid accumulation in skeletal muscle is not only due to ectopic deposition but may also be due to dysregulation of mitochondrial beta oxidation of fatty acids, both of which have been noted in the obese, diabetic state and may be causative in insulin resistance $[2,3,5]$. Indeed, skeletal muscle lipid accumulation in the form of triacylglycerols, diacylglycerols and long chain acyl-CoAs have all been negatively correlated with insulin action [7-9].
Several genetic factors have been implicated in the underlying pathophysiology of obesity and obesity-associated metabolic dysregulation of lipids and insulin resistance [10-12]; yet, the onset and progression of obesity and type 2 diabetes are largely due to environmental factors, such as increased dietary fat content and decreased physical activity levels [13]. High fat diets have long been used as a model to induce obesity, leading to skeletal muscle ectopic lipid deposition, mitochondrial dysfunction and insulin resistance [5,9,14]. Conversely, exercise training has been shown to improve skeletal muscle mitochondrial function and insulin sensitivity, while increasing beta oxidation of fatty acids [15-18]. Paradoxically, exercise has been shown to increase lipid content in skeletal muscle similar to the increase due to ectopic deposition seen during obesity $[18,19]$. Yet, metabolic derangements in skeletal muscle lead to differential levels of specific fatty acid species, suggesting that specific species of fatty acids in addition to total lipid levels may account for the detrimental or beneficial effects on insulin sensitivity [10]. In this mini review, we will discuss the role of epigenetics in mitochondrial adaptations in obesity and insulin resistance and their relation to lipid metabolism.

\section{Mitochondrial Dysfunction and Epigenetics}

Epigenetic regulation of gene expression, or alterations in gene expression that occur independent of changes in the DNA sequence, include modifications to the chromatin structure, such as DNA methylation and covalent modifications to histone proteins including acetylation, phosphorylation, sumoylation, methylation, ADP ribosylation and ubiquitination $[8,20]$. Gene expression changes result in various physiological states, contributing to the onset and progression of obesity and type 2 diabetes $[1,21]$. High fat diets have been shown to alter the epigenome in association with obesity and type 2 diabetes. For example, in utero, high fat diet feeding and maternal obesity alters DNA methylation patterns and histone modifications while increasing susceptibility to obesity in offspring of high fat diet-fed dams and high fat diet feeding alters DNA methylation in skeletal muscle [22-25]. High fat diet feeding has also been shown to alter DNA methylation patterns within individuals in association with differences in insulin sensitivity [26]. Additionally, obese and diabetic individuals exhibit differential DNA methylation 
patterns in skeletal muscle compared to lean individuals [27]. These epigenetic modifications occur in conjunction with altered skeletal muscle mitochondrial beta oxidation and mitochondrial number $[27,28]$. Exercise training has also been shown to alter epigenetic modifications in association with insulin sensitivity and improvements in adiposity and mitochondrial function [28]. Interestingly, genes that are epigenetically regulated by fatty acids and exercise are also important regulators of mitochondrial adaptations, such as changes in fatty acid beta oxidation capacity and mitochondrial number $[27,28]$. Collectively, these studies show that skeletal muscle mitochondrial adaptations, which contribute to lipid metabolism through regulation of fatty acid oxidation, may be epigenetically regulated by diet and exercise.

A major role of mitochondria is to produce ATP, and this is accomplished partially through beta oxidation of fatty acids which also primarily occurs within the mitochondrion. During beta oxidation, fatty acids transported into the mitochondrion are oxidized such that their chain length is shortened by two carbons with each round of oxidation until the final product, acyl-coA is produced. An initiating step in mitochondrial beta oxidation is transport of the fatty acid into the mitochondrion. For long-chain acyl-CoAs, which are unable to diffuse from the cytosol into the mitochondria, this is accomplished by addition of carnitine, carried out by the mitochondrial carnitine shuttle and resulting in the formation of specific acylcarnitine species [29]. Medium and short chain acyl-coAs can cross the mitochondrial membranes independent of the carnitine shuttle [30]. Dietary changes can inhibit the initial step in the mitochondrial carnitine shuttle leading to high rates of incomplete fat oxidation and lead to production of specific acylcarnitine species resulting from excess fatty acid byproducts [31].

Production of acylcarnitine from acyl-coAs, produced as intermediates and by- and end-products during fatty acid oxidation, aids in the intracellular transport of fatty acids and may serve as an indicator of mitochondrial function in regards to lipid metabolism $[14,31]$. Increased levels of long and medium chain acylcarnitines, including octadecenoylcarnitine (C18:1), tetradecenoylcarnitine (C14:1), tetradecadienylcarnitine(C14:2)anddecreasesinshortchain acylcarnitines, such as malonylcarnitine/hydroxybutyrylcarnitine $(\mathrm{C} 3 \mathrm{DC}+\mathrm{C} 4 \mathrm{OH})$, have been noted in the diabetic and prediabetic states [32].

\section{Fatty Acids and Epigenetic Regulation}

Interestingly, several fatty acid species have been shown to play a role in epigenetic regulation of gene expression. Most notably, short chain fatty acids such as those produced during fermentation of dietary fiber and as byproducts of fatty acid oxidation are known to inhibit the enzyme histone deacetylase (HDAC), an enzyme important in removing acetyl groups from histone proteins [33]. Butyrate, acetate, propionate, valerate, and capropate have all been shown to inhibit HDAC, therefore allowing hyperacetylation of core histone proteins [34]. In addition to epigenetically regulating histone acetylation patterns, short chain fatty acids also alter DNA methylation patterns [35]. Thus, the epigenetic effects of fatty acid oxidation byproducts appear to be pleiotropic in their epigenetic actions. Not only do short chain fatty acids play a role in altering epigenetic modifications; but other metabolic byproducts, such as pyruvate and lactate, have also been shown to inhibit HDAC activity to epigenetically regulate gene expression $[1,7]$. More recently, short chain fatty acids, such as butyrate, have been shown to act as anti-obesogenic and anti-diabetic agents $[9,12]$. The antiobesogenic and anti-diabetic effects of these short chain fatty acids may be due partially to the upregulation of mitochondrial function [13], more specifically upregulation of skeletal muscle mitochondrial fatty acid oxidation and energy expenditure [36].

In contrast to HDAC, which catalyzes the deacetylation of histones, the enzyme histone acetylase (HAT) catalyzes the acetylation of histone proteins to epigenetically regulate gene expression [11]. The major HAT cofactor necessary for histone acetylation is acetyl-coA, the by- and end-product of mitochondrial beta oxidation and by-product of glucose oxidation [37]. Cellular supply of acetyl-coA is a rate limiting step in the acetylation of histone proteins and the epigenetic regulation of gene expression [38]. Thus, metabolism, including that of fatty acids, is tightly associated with epigenetic modifications and gene regulation through at least two separate mechanisms, direct inhibition of HDAC and stimulation of HAT activity through cofactor availability.

\section{Conclusion}

Tight regulation of metabolism in response to environmental stimuli, such as diet and exercise, appear to be regulated through an epigenetic mechanism involving metabolic byproducts and mitochondrial function [39]. Mitochondrial function in skeletal

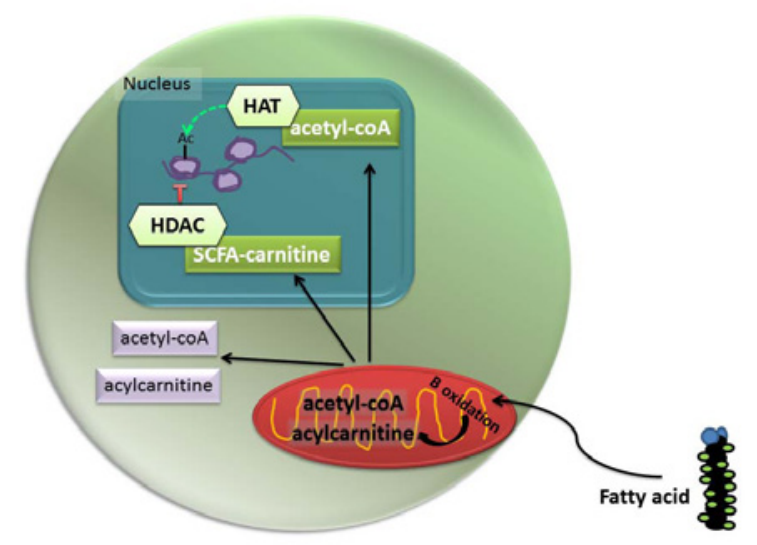

Figure 1: Model of mitochondrial-derived fatty acid epigenetic regulation. Fatty acids undergo mitochondrial beta oxidation, producing byproducts and the final beta oxidation endproduct, acetyl-coA. Byproducts of beta oxidation, including the short chain fatty acids (SCFA) butyrate, acetate, propionate, valerate and capropate, may be conjugated to carnitine and translocate intracellularly to the cytoplasm and nucleus. Translocated fatty acids may provide pools of acetyl-coA for histone acetylation by histone acetyltransferase (HAT) or may directly inhibit histone deacetylation by histone deacetylase (HDAC) in order to epigenetically regulate gene expression and subsequent phenotypes and physiologies. 
muscle plays an important role in determining insulin resistance through oxidation of fatty acids, during which specific species of fatty acids are produced as metabolic byproducts [2]. These byproducts can be transported intracellularly as acylcarnitines and have been reported to reside in the nucleus $[34,40]$. Moreover, short chain fatty acids specifically are known to regulate epigenetic modifications and gene expression. Based on the current literature, we propose a model (shown in Figure 1) whereby diet and exercise regulate mitochondrial function to produce specific fatty acid byproducts from beta oxidation and these byproducts translocate to the nucleus to provide the impetus for epigenetic regulation of gene expression leading to adaptations in phenotype and physiology to environmental conditions.

\section{Acknowledgements}

The Henagan lab is supported by NIH NCCAM (5P50AT002776-09; TMH), NIH Indiana CTSI Core Pilot Grant (UL1 TR001108; TMH), Purdue University Ralph W. and Grace M. Showalter Research Trust and Showalter Bioinformatics Grant (TMH).

\section{References}

1. Drong AW, Lindgren CM, McCarthy MI. The Genetic and Epigenetic Basis of Type 2 Diabetes and Obesity. Clin Pharmacol Ther. 2012 ; 92(6):707-715. doi: 10.1038/clpt.2012.149.

2. Bajpeyi S, Myrland CK, Covington JD, Obanda D, Cefalu WT, Smith SR, et al. Lipid in skeletal muscle myotubes is associated to the donors' insulin sensitivity and physical activity phenotypes. Obesity (Silver Spring). 2014; 22(2):426-34. doi: 10.1002/oby.20556.

3. Kelley DE, He J, Menshikova EV, Ritov VB. Dysfunction of Mitochondria in Human Skeletal Muscle in Type 2 Diabetes. Diabetes. 2002; 51(10):2944-2950

4. Koves TR, Ussher JR, Noland RC, Slentz D, Mosedale M, Ilkayeva O, et al. Mitochondrial Overload and Incomplete Fatty Acid Oxidation Contribute to Skeletal Muscle Insulin Resistance. Cell Metab. 2008; 7(1):45-56. doi: 10.1016/j.cmet.2007.10.013.

5. Sparks LM, Xie H, Koza RA, Mynatt R, Hulver MW, Bray GA, et al. A High-Fat Diet Coordinately Downregulates Genes Required for Mitochondrial Oxidative Phosphorylation in Skeletal Muscle. Diabetes. 2005; 54(7):1926-1933.

6. DeFronzo RA, Tripathy D. Skeletal Muscle Insulin Resistance Is the Primary Defect in Type 2 Diabetes. Diabetes Care. 2009; 32 Suppl 2:S157-63. doi: 10.2337/dc09-S302.

7. Ellis BA, Poynten A, Lowy AJ, Furler SM, Chisholm DJ, Kraegen EW, et al. Long-chain acyl-CoA esters as indicators of lipid metabolism and insulin sensitivity in rat and human muscle. Am J Physiol Endocrinol Metab. 2000; 279(3):E554-560.

8. Pan DA, Lillioja S, Kriketos AD, Milner MR, Baur LA, Bogardus C, et al. Skeletal Muscle Triglyceride Levels Are Inversely Related to Insulin Action. Diabetes. 1997; 46(6):983-988.

9. Itani SI, Ruderman NB, Schmieder F, Boden G. Lipid-induced insulin resistance in human muscle is associated with changes in diacylglycerol, protein kinase C, and IkappaB-alpha. Diabetes. 2002; 51(7):2005-2011.

10. Makowski L, Noland RC, Koves TR, Xing W, Ilkayeva OR, Muehlbauer MJ, et al. Metabolic profiling of PPARalpha-/- mice reveals defects in carnitine and amino acid homeostasis that are partially reversed by oral carnitine supplementation. FASEB J. 2009; 23(2):586-604. doi: 10.1096/fj.08-119420.

11. Roth SY, Denu JM, Allis CD. Histone Acetyltransferases. Annu Rev Biochem. 2001; 70(1):81-120.

12. Perry JR, Voight BF, Yengo L, Amin N, Dupuis J, Ganser M, et al. Stratifying Type 2 Diabetes Cases by BMI Identifies Genetic Risk Variants in LAMA1 and Enrichment for Risk Variants in Lean Compared to Obese Cases. PLoS Genet. 2012; 8(5):e1002741. doi: 10.1371/journal.pgen.1002741.

13. Hu FB, Manson JE, Stampfer MJ, Colditz G, Liu S, Solomon CG, et al. Diet, Lifestyle and the Risk of Type 2 Diabetes Mellitus in Women. N Engl J Med. 2001; 345(11):790-797.

14. Henagan TM, Lenard NR, Gettys TW, Stewart LK. Dietary Quercetin Supplementation in Mice Increases Skeletal Muscle PGC1 $\alpha$ Expression, Improves Mitochondrial Function and Attenuates Insulin Resistance in a Time-Specific Manner. PLoS One. 2014; 9(2):e89365. doi: 10.1371/ journal.pone.0089365.

15. Solomon TP, Sistrun SN, Krishnan RK, Del Aguila LF, Marchetti CM, 0 'Carroll SM, et al. Exercise and diet enhance fat oxidation and reduce insulin resistance in older obese adults. J Appl Physiol (1985). 2008; 104(5):1313-1319. doi: 10.1152/japplphysiol.00890.2007.

16. Van Tienen FH, Praet SF, de Feyter HM, van den Broek NM, Lindsey PJ, Schoonderwoerd KG, et al. Physical Activity Is the Key Determinant of Skeletal Muscle Mitochondrial Function in Type 2 Diabetes. J Clin Endocrinol Metab. 2012; 97(9):3261-3269. doi: 10.1210/jc.20113454.

17. Galgani JE, Johannsen NM, Bajpeyi S, Costford SR, Zhang Z, Gupta AK, et al. Role of Skeletal Muscle Mitochondrial Density on ExerciseStimulated Lipid Oxidation. Obesity (Silver Spring). 2012; 20(7):138793. doi: 10.1038/oby.2011.166.

18. Meex RC, Schrauwen-Hinderling VB, Moonen-Kornips E, Schaart G, Mensink M, Phielix E, et al. Restoration of Muscle Mitochondrial Function and Metabolic Flexibility in Type 2 Diabetes by Exercise Training Is Paralleled by Increased Myocellular Fat Storage and Improved Insulin Sensitivity. Diabetes. 2010; 59(3):572-579. doi: $10.2337 / \mathrm{db} 09-1322$.

19. Schenk S, Horowitz JF. Acute exercise increases triglyceride synthesis in skeletal muscle and prevents fatty acid-induced insulin resistance. J Clin Invest. 2007; 117(6):1690-1698.

20.Zhou VW, Goren A, Bernstein BE. Charting histone modifications and the functional organization of mammalian genomes. Nat Rev Genet. 2011; 12(1):7-18. doi: 10.1038/nrg2905.

21. Milagro FI, Campión J, García-Díaz DF, Goyenechea E, Paternain L, Martínez JA. High fat diet-induced obesity modifies the methylation pattern of leptin promoter in rats. J Physiol Biochem. 2009; 65(1):1-9.

22. Dudley KJ, Sloboda DM, Connor KL, Beltrand J, Vickers MH. Offspring of Mothers Fed a High Fat Diet Display Hepatic Cell Cycle Inhibition and Associated Changes in Gene Expression and DNA Methylation. PLoS One. 2011; 6(7):e21662. doi: 10.1371/journal.pone.0021662.

23. Ge ZJ, Luo SM, Lin F, Liang QX, Huang L, Wei YC, et al. DNA Methylation in Oocytes and Liver of Female Mice and Their Offspring: Effects of High-Fat-Diet-Induced Obesity. Environ Health Perspect. 2014; 122(2):159-164. doi: 10.1289/ehp.1307047.

24. Suter M, Bocock P, Showalter L, Hu M, Shope C, McKnight R, et al. Epigenomics: maternal high-fat diet exposure in utero disrupts peripheral circadian gene expression in nonhuman primates. FASEB 


\section{J. 2011; 25(2):714-726. doi: 10.1096/fj.10-172080.}

25. Jacobsen SC, Brøns C, Bork-Jensen J, Ribel-Madsen R, Yang B, Lara E et al. Effects of short-term high-fat overfeeding on genome-wide DNA methylation in the skeletal muscle of healthy young men. Diabetologia. 2012; 55(12):3341-3349. doi: 10.1007/s00125-012-2717-8.

26. Khalyfa A, Carreras A, Hakim F, Cunningham JM, Wang Y, Gozal D. Effects of late gestational high-fat diet on body weight, metabolic regulation and adipokine expression in offspring. Int J Obes (Lond) 2013; 37(11):1481-1489. doi: 10.1038/ijo.2013.12.

27. Barrès R, Osler ME, Yan J, Rune A, Fritz T, Caidahl K, et al. Non-CpG Methylation of the PGC- $1 \alpha$ Promoter through DNMT3B Controls Mitochondrial Density. Cell Metab. 2009; 10(3):189-198. doi: 10.1016/j.cmet.2009.07.011.

28. Barrès R, Yan J, Egan B, Treebak JT, Rasmussen M, Fritz T, et al. Acute Exercise Remodels Promoter Methylation in Human Skeletal Muscle. Cell Metab. 2012; 15(3):405-411. doi: 10.1016/j.cmet.2012.01.001.

29. Kim JY, Koves TR, Yu GS, Gulick T, Cortright RN, Dohm GL, et al. Evidence of a malonyl-CoA-insensitive carnitine palmitoyltransferase I activity in red skeletal muscle. Am J Physiol Endocrinol Metab. 2002; 282(5):E1014-1022.

30. Violante S, Ijlst L, Te Brinke H, Tavares de Almeida I, Wanders RJ, Ventura FV, et al. Carnitine palmitoyltransferase 2 and carnitine/ acylcarnitine translocase are involved in the mitochondrial synthesis and export of acylcarnitines. FASEB J. 2013; 27(5):2039-2044. doi: 10.1096/fj.12-216689.

31. Noland RC, Koves TR, Seiler SE, Lum H, Lust RM, Ilkayeva O, et al. Carnitine Insufficiency Caused by Aging and Overnutrition Compromises Mitochondrial Performance and Metabolic Control. J Biol Chem. 2009; 284(34):22840-22852. doi: 10.1074/jbc.M109.032888.

32. Mai M, Tönjes A, Kovacs P, Stumvoll M, Fiedler GM, Leichtle AB. Serum Levels of Acylcarnitines Are Altered in Prediabetic Conditions. PLoS One. 2013; 8(12):e82459. doi: 10.1371/journal.pone.0082459.
33. Waldecker M, Kautenburger T, Daumann H, Busch C, Schrenk D. Inhibition of histone-deacetylase activity by short-chain fatty acids and some polyphenol metabolites formed in the colon. J Nutr Biochem. 2008; 19(9):587-593.

34. Hinnebusch BF, Meng S, Wu JT, Archer SY, Hodin RA. The Effects of Short-Chain Fatty Acids on Human Colon Cancer Cell Phenotype Are Associated with Histone Hyperacetylation. J Nutr. 2002; 132(5):10121017.

35. Boffa LC, Mariani MR, Parker MI. Selective Hypermethylation of Transcribed Nucleosomal DNA by Sodium Butyrate. Exp Cell Res. 1994; 211(2):420-423.

36. Gao Z, Yin J, Zhang J, Ward RE, Martin RJ, Lefevre M, et al. Butyrate Improves Insulin Sensitivity and Increases Energy Expenditure in Mice. Diabetes. 2009; 58(7):1509-1517. doi: 10.2337/db08-1637.

37. Takahashi H, McCaffery JM, Irizarry RA, Boeke JD. Nucleocytosolic Acetyl-Coenzyme A Synthetase Is Required for Histone Acetylation and Global Transcription. Mol Cell. 2006; 23(2):207-217.

38. Morrish F, Noonan J, Perez-Olsen C, Gafken PR, Fitzgibbon M, Kelleher J, et al. Myc-dependent Mitochondrial Generation of AcetylCoA Contributes to Fatty Acid Biosynthesis and Histone Acetylation during Cell Cycle Entry. J Biol Chem. 2010; 285(47):36267-36274. doi: 10.1074/jbc.M110.141606

39. Galdieri L, Vancura A. Acetyl-CoA Carboxylase Regulates Global Histone Acetylation. J Biol Chem. 2012; 287(28): 23865-23876. doi: 10.1074/jbc.M112.380519.

40. Huang H, Starodub O, McIntosh A, Kier AB, Schroeder F. Liver fatty acid binding protein targets fatty acids to the nucleus: real-time confocal and multiphoton fluorescence imaging in living cells. J Biol Chem. 2002; 277(32):29139-29151. 